\title{
Pro: Propofol is a Better Induction Agent than Etomidate
}

\author{
Naresh Aggarwal ${ }^{1}$ \\ ${ }^{1}$ Department of Cardiac Anaesthesia, Manipal Hospital, Delhi, India
}

J Card Crit Care:2021;5:70-71
Address for correspondence Naresh Aggarwal, MD, Department of Cardiac Anaesthesia, Manipal Hospital, Dwarka, New Delhi 110029, India (e-mail: Naresh15@gmail.com).

\begin{abstract}
Keywords

- etomidate

- induction agent

- propofol

Induction refers to the start of anesthesia when the patient is rendered unconscious. The intravenous induction agents allow the patient to experience a pleasant loss of consciousness while also rapidly achieving surgical levels of anesthesia. An ideal induction agent must have a rapid onset and offset of action, and must be easy to administer and without significant side effects. Currently, the commonest used agents include sodium pentothal, propofol, ketamine, and etomidate. Unfortunately, none of these agents possess the characteristics of an ideal induction agent. In this article, we will discuss the merits of propofol as an ideal induction agent for cardiac surgery and how it scores over etomidate for the same purpose.
\end{abstract}

\section{Introduction}

Induction refers to the start of anesthesia when the patient is rendered unconscious. The intravenous (IV) induction agents allow the patient to experience a pleasant loss of consciousness while also rapidly achieving surgical levels of anesthesia. An ideal induction agent must have a rapid onset and offset of action, and must be easy to administer and without significant side effects. Currently, the commonest used agents include sodium pentothal, propofol, ketamine, and etomidate. Unfortunately, none of these agents possess the characteristics of an ideal induction agent. In this article, we will discuss the merits of propofol as an ideal induction agent for cardiac surgery and how it scores over etomidate for the same purpose.

\section{General Characteristics of Propofol}

Propofol is a unique compound compared with other IV agents. It is a simple phenol substituted with two isopropyl groups in each of the position adjacent to the hydroxyl groups. It is formulated as an emulsion of a soybean oil/propofol mixture in water. The currently available preparation is $1 \%$ propofol, $10 \%$ soybean oil, $1.2 \%$ purified egg phospholipid as

published online March 23, 2021
Journal of Cardiac Critical Care TSS xxxx;xxx:xxx:xxx an emulsifier, $2.25 \%$ glycerol as a tonicity-adjusting agent, and sodium hydroxide to adjust the $\mathrm{pH}$. Propofol formulations contain EDTA, sodium metabisulfite, or benzyl alcohol as antimicrobial agents. Fospropofol is a recently marketed water-soluble prodrug that is broken down by alkaline phosphatase. It does not produce pain of IV injection. It is used as a hypnotic, sedative, and antiemetic. Propofol is frequently used as a sole agent or in combination with other drugs for total intravenous anesthesia.

\section{Physiological Properties}

The most prominent effect of propofol is a decrease in arterial blood pressure during induction accompanied by a decrease in cardiac output/cardiac index, stroke volume index, and systemic vascular resistance. In valvular heart disease, pulmonary artery and pulmonary capillary wedge pressure also are reduced. Heart rate does not significantly change with an induction dose of propofol. The vasodilatory and myocardial depressant effects are concentration-dependent. Propofol can decrease the tidal volume and respiratory rate, which can lead to apnea depending on the dose, speed of injection, and concomitant premedication. It is also a very potent bronchodilator and can be used safely in patients suffering
(C)2021. Official Publication of The Simulation Society (TSS), accredited by International Society of Cardiovascular Ultrasound (ISCU).

This is an open access article published by Thieme under the terms of the Creative Commons Attribution-NonDerivative-NonCommercial-License, permitting copying and reproduction so long as the original work is given appropriate credit. Contents may not be used for commercial purposes, or adapted, remixed, transformed or built upon. (https://creativecommons.org/licenses/by-nc-nd/4.0/).

Thieme Medical and Scientific Publishers Pvt. Ltd. A-12, 2nd Floor, Sector 2, Noida-201301 UP, India 
from asthma. It leads to rapid onset of hypnosis and amnesia. Propofol possesses anticonvulsant properties and can be used to treat epileptic seizures despite its propensity to precipitate grand mal seizure.

\section{How Does Propofol Fare When Compared with Other Agents?}

A randomized trial compared the effects of propofol with thiopentone and etomidate on respiratory resistance following endotracheal intubation. It was demonstrated that propofol lowered the resistance to a greater extent than thiopentone and etomidate. ${ }^{1}$ A combination of propofol and etomidate was found to be better than either propofol or etomidate alone when used for induction during general anesthesia. ${ }^{2}$ The primary concern with the use of propofol is hypotension. It was shown that propofol causes a 34\% reduction in mean arterial pressure-time integral from the baseline after induction when compared with etomidate, despite the use of vasopressors. ${ }^{3}$

\section{Is Etomidate All that Safe?}

Etomidate is much more potent as an inhibitor of adrenal steroid synthesis than as an induction agent. A single dose of etomidate is sufficient enough to suppress the adrenal function for 24 to 48 hours and is currently under investigation in clinical trials as a therapeutic agent for poorly controlled Cushing's syndrome. It suppresses adrenal function by inhibition of 11- $\square$ hyroxylation. ${ }^{4}$ Anesthesiologists using etomidate must be aware of this potential side-effect and be ready with parenteral corticosteroids if the need arises during the perioperative period. ${ }^{5}$

\section{Indications for Propofol in Cardiac Surgery}

Following are the indications for propofol in cardiac surgery:

- Normotensive patients with good ventricular function.

- Hypertensive patients.

- Malignant hyperthermia.

- Induction and maintenance of general anesthesia.

- Total IV anesthesia (TIVA).

- Cardiac catheterization procedures.

\section{Propofol versus Etomidate in Cardiac Surgery-Who Wins?}

It has been proven beyond doubt that cardiac surgery is a potent stimulator of inflammatory response. The cortisol which is secreted during cardiopulmonary bypass is vital for the maintenance of vascular tone and inhibition of nitric oxide synthesis. Since cortisol is thought to act synergistically with endogenous vasopressors, the impairment of these stress hormones is likely to contribute to the increase in the vasopressor requirements in the postoperative period.

Cortisol also aids in the maintenance of vascular permeability, thereby possibly decreasing ischemia/reperfusion injury. This highlights the significance of steroids in maintenance of hemodynamic stability in the perioperative period. ${ }^{6}$

Since etomidate suppresses adrenal glands, thereby preventing the release of corticosteroids, its use in cardiac surgery must not be taken lightly. Propofol on the other hand does not inhibit adrenal function and when used judiciously with opioids can provide a good hemodynamic control during induction.

\section{Conclusions}

Etomidate has a superior cardiovascular stability without doubt. However, it is not without adverse effects such as pain on injection, an increased incidence of postoperative vomiting, hiccups, and myoclonus. The incidence of emergence delirium incidence is higher in the etomidate group. Perhaps unsurprisingly, there have been no studies showing superior outcomes with etomidate compared with other general anesthetics, it appears that the judicious use of other induction agents is warranted.

\section{Conflict of Interest}

None declared.

\section{References}

1 Eames WO, Rooke GA, Wu RS, Bishop MJ. Comparison of the effects of etomidate, propofol, and thiopental on respiratory resistance after tracheal intubation. Anesthesiology 1996;84(6):1307-1311

2 Meena K, Meena R, Nayak SS, Prakash S, Kumar A. A comparative study of effect of propofol, etomidate and propofol plus etomidate induction on hemodynamic response to endotracheal intubation: a RCT. J Anesth Clin Res 2016;7(5):622

3 Hannam JA, Mitchell SJ, Cumin D, et al. Haemodynamic profiles of etomidate vs propofol for induction of anaesthesia: a randomised controlled trial in patients undergoing cardiac surgery. Br J Anaesth 2019;122(2):198-205

4 de Jong FH, Mallios C, Jansen C, Scheck PA, Lamberts SW. Etomidate suppresses adrenocortical function by inhibition of 11 beta-hydroxylation. J Clin Endocrinol Metab 1984;59(6):1143-1147

5 Wagner RL, White PF. Etomidate inhibits adrenocortical function in surgical patients. Anesthesiology 1984;61(6):647-651

6 Iribarren JL, Jiménez JJ, Hernández D, et al. Relative adrenal insufficiency and hemodynamic status in cardiopulmonary bypass surgery patients. A prospective cohort study. J Cardiothorac Surg 2010;5:26 